\title{
Simultaneous Coverage and Tracking (SCAT) of Moving Targets with Robot Networks
}

\author{
Luciano C. A. Pimenta, Mac Schwager, Quentin Lindsey, Vijay Kumar, Daniela \\ Rus, Renato C. Mesquita, and Guilherme A. S. Pereira
}

\begin{abstract}
We address the problem of simultaneously covering an environment and tracking intruders (SCAT). The problem is translated to the task of covering environments with time-varying density functions under the locational optimization framework. This allows for coupling the basic subtasks: task assignment, coverage, and tracking. A decentralized controller with guaranteed exponential convergence is devised. The SCAT algorithm is verified in simulations and on a team of robots.
\end{abstract}

\section{Introduction}

Three important problems in the field of cooperative robotics are: (i) environment coverage, (ii) task assignment, and (iii) target (intruder) tracking. These three problems are the basic subtasks that must be solved in the global problem of tracking multiple intruders in a pre-specified region. When the number of intruders and their locations is unknown, the need for environment coverage is important to maximize the probability of detecting them. Task assignment is necessary to define when a given agent should perform a coverage behavior and when it should do intruder tracking. For multiple intruders it is also necessary to allocate intruders to tracking agents. After assigning an intruder-tracking task to an agent, it is desirable to use control laws with guaranteed convergence.

Luciano Pimenta, Quentin Lindsey, and Vijay Kumar

GRASP Lab, University of Pennsylvania, USA, e-mail: \{pimenta,quentinl\}@ seas.upenn.edu, kumar@grasp.upenn.edu

Mac Schwager and Daniela Rus

CSAIL, MIT, USA, e-mail: schwager@mit.edu, rus@csail.mit.edu

Luciano Pimenta, Guilherme Pereira, and Renato Mesquita

Departamento de Engenharia Elétrica, Universidade Federal de Minas Gerais, Brazil e-mail: \{lucpim,renato,gpereira\}@cpdee.ufmg.br 
A distributed and asynchronous approach for optimal coverage of a domain with identical mobile sensing agents is proposed in [3] based on a framework for optimized quantization derived in [7]. Each agent (robot) follows a gradient descent control algorithm that minimizes a functional encoding the quality of the sensing coverage. Further, this control law depends only on the information about the position of the robot and that of its immediate neighbors, which are the robots in neighboring Voronoi cells. These control laws are computed without the requirement of global synchronization. The functional also uses a distribution density function, which weights points or areas in the environment according to importance. This adaptive technique can cope with changing environments, tasks, and network topology.

Several extensions of the framework devised in [3] have been proposed. In [1] the problem of limited-range interaction between agents was addressed. In [10] different distance metrics were used to address heterogeneous sensor footprints and nonconvex environments. In addition, constraints were added to the minimization problem to deal with finite sized robots. The problem of learning the distribution density function online while moving toward the optimal locations was addressed in [11].

The most relevant paper to this work is [2], in which the authors presented the problem of tracking an intruder in time-varying environments. In this case, a dynamic target was defined and its position used in the density function computation. The problem of covering environments with time-varying density functions was addressed by using a control law where convergence guarantees were difficult to establish.

In this paper, we translate the problem of optimally covering an environment while tracking intruders into the problem of covering environments with timevarying density functions. The proposed framework allows for the coupling of the three previously mentioned basic subtasks: target assignment, coverage, and tracking in an elegant distributed manner. For the first time, a decentralized controller with guaranteed exponential convergence is also derived. Simulations and actual robots experiments are also presented to verify the proposed approach.

\section{Locational Optimization Framework}

Locational optimization addresses how to place facilities or resources to minimize some cost $[12,4]$. The canonical example is placing retail facilities to minimize the aggregate travel time of a population of customers. The locational optimization framework has been recently applied to the optimal placement of mobile sensors to provide sensor coverage of an environment [3]. In this section, we give a brief introduction to the locational optimization results relevant to our problem.

Let there be $n$ mobile sensing agents in a bounded environment represented by $Q \subset \mathbb{R}^{N}$, and the location of the $i$ th sensor is given by $\mathbf{p}_{i} \in Q$. Then $\mathbf{P}=$ $\left[\mathbf{p}_{1}^{T}, \ldots, \mathbf{p}_{n}^{T}\right]^{T} \in Q \times \ldots \times Q$ is a vector representing the configuration of the mobile 
sensor network. Consider the cost function

$$
\mathscr{H}(\mathbf{P})=\int_{Q} \min _{i \in\{1, \ldots, n\}} f\left(d\left(\mathbf{q}, \mathbf{p}_{i}\right)\right) \phi(\mathbf{q}) d \mathbf{q},
$$

where $d: \mathbb{R}^{N} \times \mathbb{R}^{N} \rightarrow \mathbb{R}_{\geq 0}$ is a function that measures distances between points $\mathbf{q} \in Q$ and sensor positions $\mathbf{p}_{i} \in Q$. The function $\phi: Q \rightarrow \mathbb{R}_{\geq 0}$ is a distribution density function, which defines a weight for each point in $Q$. The density function may reflect the probability of occurrence of events in different regions, or a measure of relative importance of different regions in $Q$. Therefore, points with greater weight values should be better covered than points with smaller values. The function $f: \mathbb{R} \rightarrow \mathbb{R}$ is a continuously differentiable, strictly increasing function over the range of $d$ that measures the degradation of sensing performance with distance. The minimum over sensors reflects the fact that a point $\mathbf{q}$ should be the responsibility of the sensor that has the best sensing performance at q. Covering the environment, $Q$, becomes an optimization to minimize the cost function (1). In the following subsection the necessary conditions to minimize the function in (1) are established, which will give the basis for a control strategy.

\subsection{Computations with Voronoi Tessellations}

Consider the minimization of (1)

$$
\min _{\mathbf{P}} \mathscr{H}(\mathbf{P})=\min _{\mathbf{P}} \int_{Q} \min _{i} f\left(d\left(\mathbf{q}, \mathbf{p}_{i}\right)\right) \phi(\mathbf{q}) d \mathbf{q} .
$$

The minimum inside the integral induces a partition of $Q$ into non-overlapping cells, $V_{i}$, to give

$$
\min _{\mathbf{P}} \mathscr{H}(\mathbf{P})=\min _{\mathbf{P}} \sum_{i=1}^{n} \int_{V_{i}} f\left(d\left(\mathbf{q}, \mathbf{p}_{i}\right)\right) \phi(\mathbf{q}) d \mathbf{q},
$$

where $V_{i}=\left\{\mathbf{q} \in Q \mid f\left(d\left(\mathbf{q}, \mathbf{p}_{i}\right)\right) \leq f\left(d\left(\mathbf{q}, \mathbf{p}_{j}\right)\right), \forall j \neq i\right\}$. Since $f$ is strictly increasing, this is equivalent to

$$
V_{i}=\left\{\mathbf{q} \in Q \mid d\left(\mathbf{q}, \mathbf{p}_{i}\right) \leq d\left(\mathbf{q}, \mathbf{p}_{j}\right), \forall j \neq i\right\}
$$

The region $V_{i}$ is the Voronoi cell of $\mathbf{p}_{i}$. The collection of Voronoi cells is called the Voronoi tessellation of $Q[9]^{1}$.

We now define a number of quantities relating to Voronoi cells. Let $\partial V_{i}$ and $\partial Q$ be the boundary of $V_{i}$ and $Q$, respectively. By $\mathbf{q}_{\partial V_{i}}(\mathbf{P})$ we mean a point $\mathbf{q} \in \partial V_{i}$, and $\mathbf{n}_{\partial V_{i}}$ is the outward facing unit normal of $\partial V_{i}$. Given a robot $i$, we define $\mathscr{N}_{i}$ as the

${ }^{1}$ It is convenient for us to use $\leq$ in the definition of Voronoi cell, rather than the more common definition with $<$ 
index set of robots that share Voronoi boundaries with $V_{i}, \mathscr{N}_{i}=\left\{j \mid V_{i} \cap V_{j} \neq \emptyset\right\}$. We denote the set of points on the Voronoi boundary shared by agents $i$ and $j$ as $l_{i j}=V_{i} \cap V_{j}$ as shown in Fig. 1. Then $\mathbf{q}_{i j}\left(\mathbf{p}_{i}, \mathbf{p}_{j}\right)$ is a point on that shared boundary, and $\mathbf{n}_{l_{i j}}$ is the unit normal of $l_{i j}$ from $\mathbf{p}_{i}$ to $\mathbf{p}_{j}$. By the definition of the Voronoi cell (3), we know the following facts:

$$
\begin{aligned}
\partial V_{i} & =\left(\cup_{j \in \mathscr{N}_{i}} l_{i j}\right) \cup\left(\partial V_{i} \cap \partial Q\right), \\
l_{i j} & =l_{j i}, \\
\mathbf{n}_{l_{i j}} & =-\mathbf{n}_{l_{j i}} .
\end{aligned}
$$

When $d$ is the Euclidean distance, the shared boundaries $l_{i j}$ are hyperplanes, and the Voronoi cells are convex.

The following lemma states an important fact about the cost function (2).

\section{Lemma 0.1 (Distributed Gradient).}

$$
\frac{\partial \mathscr{H}}{\partial \mathbf{p}_{i}}=\int_{V_{i}} \frac{\partial}{\partial \mathbf{p}_{i}} f\left(d\left(\mathbf{q}, \mathbf{p}_{i}\right)\right) \phi(\mathbf{q}) d \mathbf{q}
$$

Proof. Differentiating under the integral sign [5], we have

$$
\begin{aligned}
\frac{\partial \mathscr{H}}{\partial \mathbf{p}_{i}}= & \int_{V_{i}} \frac{\partial}{\partial \mathbf{p}_{i}} f\left(d\left(\mathbf{q}, \mathbf{p}_{i}\right)\right) \phi(\mathbf{q}) d \mathbf{q} \\
& +\int_{\partial V_{i} \cap \partial Q} f\left(d\left(\mathbf{q}, \mathbf{p}_{i}\right)\right) \phi(\mathbf{q}) \frac{\partial \mathbf{q}_{\partial V_{i}}(\mathbf{P})}{\partial \mathbf{p}_{i}} \mathbf{n}_{\partial V_{i}} d \mathbf{q} \\
& +\sum_{j \in \mathscr{N}_{i}} \int_{l_{i j}}\left(f\left(d\left(\mathbf{q}, \mathbf{p}_{i}\right)-f\left(d\left(\mathbf{q}, \mathbf{p}_{j}\right)\right)\right) \phi(\mathbf{q}) \frac{\partial \mathbf{q}_{l_{i j}}\left(\mathbf{p}_{i}, \mathbf{p}_{j}\right)}{\partial \mathbf{p}_{i}} \mathbf{n}_{l_{i j}} d \mathbf{q}\right.
\end{aligned}
$$

where $\frac{\partial \mathbf{q}_{\partial V_{i}}(\mathbf{P})}{\partial \mathbf{p}_{i}}$ and $\frac{\partial \mathbf{q}_{l j i}\left(\mathbf{p}_{i}, \mathbf{p}_{j}\right)}{\partial \mathbf{p}_{i}}$ are $N \times N$ matrices. By definition of $l_{i j}, d\left(\mathbf{q}, \mathbf{p}_{i}\right)=$ $d\left(\mathbf{q}, \mathbf{p}_{j}\right) \forall \mathbf{q} \in l_{i j}$, so the last sum vanishes. Since points on the boundary of the environment do not change position as a function of $\mathbf{p}_{i}$, we have

$$
\frac{\partial \mathbf{q}_{\partial V_{i}}}{\partial \mathbf{p}_{i}}=0 \quad \forall \mathbf{q} \in \partial V_{i} \cap \partial Q
$$

and the second term vanishes.

Lemma 0.1 means that the gradient

$$
\frac{\partial \mathscr{H}}{\partial \mathbf{P}}=\left[\begin{array}{ll}
\frac{\partial \mathscr{H}^{T}}{\partial \mathbf{p}_{i}} & \cdots
\end{array}\right]^{T}
$$

is distributed among the agents in the sense that an agent $i$ can compute its own gradient component, $\partial \mathscr{H} / \partial \mathbf{p}_{i}$, using only information relevant to its Voronoi cell. This allows the design of distributed gradient algorithms, as was shown in [3]. 


\subsection{Euclidean Setting}

For the remainder of the paper, we will restrict ourselves to agents living on a plane $(N=2)$, where $d$ is the Euclidean distance, $f(x)=x^{2}$, and the environment $Q$ is convex. For a discussion of controllers for the general setting, please see [10]. In this restricted setting, the cost function becomes

$$
\mathscr{H}=\sum_{i=1}^{n} \int_{V_{i}}\left\|\mathbf{q}-\mathbf{p}_{i}\right\|^{2} \phi(\mathbf{q}) d \mathbf{q} .
$$

Define the quantities

$$
M_{V_{i}}=\int_{V_{i}} \phi(\mathbf{q}) d \mathbf{q}, \mathbf{L}_{V_{i}}=\int_{V_{i}} \mathbf{q} \phi(\mathbf{q}) d \mathbf{q}, \text { and } \mathbf{C}_{V_{i}}=\mathbf{L}_{V_{i}} / M_{V_{i}}
$$

Then Lemma 0.1 simplifies to

$$
\frac{\partial \mathscr{H}}{\partial \mathbf{p}_{i}}=-\int_{V_{i}} 2\left(\mathbf{q}-\mathbf{p}_{i}\right) \phi(\mathbf{q}) d \mathbf{q}=-2 M_{V_{i}}\left(\mathbf{C}_{V_{i}}-\mathbf{p}_{i}\right) .
$$

Clearly, critical points of $\mathscr{H}$ (configurations where the gradient is zero) are those in which every agent is at the centroid of its Voronoi cell, $\mathbf{p}_{i}=\mathbf{C}_{V_{i}} \forall i$. The resulting partition of the environment is commonly called a Centroidal Voronoi Tessellation (CVT). It is known that the critical points are local minima of $\mathscr{H}^{2}$. Therefore, we desire that every agent drives to the generalized centroid of its Voronoi region to locally minimize the cost function (7). We do not attempt to find global optima as that is known to be difficult (NP-hard for a given discretization of $Q$ ) even in the fully centralized case. Next, we present a distributed control law that is used in [3] to converge to a CVT.

\subsection{Continuous-Time Lloyd Algorithm}

A classic discrete-time method to compute CVTs is Lloyd's algorithm [7]. In each iteration this method executes three steps: (i) compute the Voronoi regions; (ii) compute the centroids; (iii) move each point site to the corresponding centroid.

In [3] a continuous-time version of this approach is proposed for kinematic models

$$
\dot{\mathbf{p}}_{i}=\mathbf{u}_{i}
$$

The control law

$$
\mathbf{u}_{i}=\gamma\left(\mathbf{C}_{V_{i}}-\mathbf{p}_{i}\right)
$$

guarantees that the system converges to a CVT, where $\gamma$ is a positive gain. This control law is a gradient-descent approach $\left(\mathbf{u}_{i} \propto-\partial \mathscr{H} / \partial \mathbf{p}_{i}\right.$ from (9)).

\footnotetext{
${ }^{2}$ To prove this, one may consider the positive definiteness of the Hessian of $\mathscr{H}$ at such points.
} 


\section{Tracking Moving Targets}

Consider the time-dependent coverage function

$$
\mathscr{H}(\mathbf{P}, t)=\sum_{i=1}^{n} \int_{V_{i}}\left\|\mathbf{q}-\mathbf{p}_{i}\right\|^{2} \phi(\mathbf{q}, t) d \mathbf{q} .
$$

More specifically, we are interested in tracking targets that invade a given region $Q$. In our approach, we do not limit the number of intruders. Furthermore, the location where they will appear and the instant of time of invasion are unknown. Therefore, we define the problem of tracking intruders while also keeping uniform coverage of $Q$. The uniform coverage is important to increase the probability of detecting new intruders. In order to provide a fully decentralized approach, the assignment of tasks should also be decentralized. There are $l(t)+1$ tasks, where $l(t)$ is the number of intruders at a given time $t,(l(t)$ tracking tasks and 1 uniform coverage task) to be accomplished. The number of tracking tasks, $l(t)$, can change over time as intruders enter and leave the environment. An intruder is only tracked while it is inside the environment $Q$.

We assume that each agent, $i$, is able to estimate the position and velocity of an intruder that is located inside the corresponding Voronoi cell $V_{i}$. In addition, we assume that the position and velocity of an intruder that is located at a distance $R_{t}$ from the Voronoi cell $V_{i}$ can be obtained by means of estimation or communication. This tuning parameter, $R_{t}$, represents the maximum tracking distance. This means that intruders located at distances greater than $R_{t}$ from $V_{i}$ will not be considered for the control law of agent $i$. In other words, the task of tracking this intruder will not be assigned to $i$.

We model the task assignment problem by composing radial basis functions, $\phi_{i}$, which represent each intruder. Thus, we define the time-varying density function

$$
\phi(\mathbf{q}, t)=\sum_{i=1}^{l} \alpha_{i} \phi_{i}(\mathbf{q}, t)+\beta,
$$

where $\alpha_{i}$ and $\beta$ are positive tuning constants that define the importance of a given task. The parameter $\alpha_{i}$ defines the importance of tracking intruder $i$, while the parameter $\beta$ defines the importance of uniform coverage.

We consider radial basis functions that are centered at the intruder position, reach a maximum value at this position, and decreases to a negligible value at a distance $R_{t}$ from the center. Therefore, the local computation of $\phi$, performed by agent $i$, considers only the intruders within tracking distance. An option is the Gaussian function in [2]:

$$
\phi_{i}(\mathbf{q}, t)=A \exp \left(-\frac{\left(x-x_{i}(t)\right)^{2}}{2 \sigma^{2}}-\frac{\left(y-y_{i}(t)\right)^{2}}{2 \sigma^{2}}\right)
$$


In this case, $R_{t}$ is related to the value of $\sigma$. We let $R_{t}=\lambda \sigma$, where $\lambda$ is a positive constant such that the values of $\phi$ at points out of this $\lambda \sigma$ range can be neglected.

One could also think of a probabilistic model of intruders and also use Gaussian density functions which could even have a different standard deviation for each axis. Such a modelling and procedures to find optimal tuning parameters $\alpha_{i}, \beta, A$, and $\sigma$ are out of the scope of this paper. In the next subsection we discuss a decentralized controller that minimizes $\mathscr{H}(\mathbf{P}, t)$.

\subsection{Exponential Controller}

The optimal deployment occurs when each agent is located at its Voronoi centroid, which is computed according to (8), (see section 2.1). Optimal deployment for timevarying density functions requires each agent to track its corresponding centroid over time. If the centroids were perfectly tracked, we would be able to guarantee that the function in (12) remains at a local minimum. Based on this, we define the performance function

$$
E=\sum_{i=1}^{n} M_{V_{i}}\left\|\mathbf{C}_{V_{i}}-\mathbf{p}_{i}\right\|^{2}
$$

which yields a controller with exponential performance.

To maintain or improve tracking of the centroid according to the function in (15), we desire $\dot{E} \leq 0, \forall t$. Computing the time derivative leads to

$$
\dot{E}=\sum_{i=1}^{n}\left(\frac{\partial E^{T}}{\partial \mathbf{p}_{i}} \dot{\mathbf{p}}_{i}+F_{i}\right)
$$

where

$$
F_{i}=\left(\mathbf{C}_{V_{i}}-\mathbf{p}_{i}\right)^{T} \int_{V_{i}}\left(2 \mathbf{q}-\mathbf{C}_{V_{i}}-\mathbf{p}_{i}\right) \dot{\phi}(\mathbf{q}, t) d \mathbf{q}
$$

is the term resulting from the fact that $\phi(\mathbf{q}, t)$ is time varying. After some algebraic effort, we can calculate the partial derivatives of $E$ as

$$
\frac{\partial E}{\partial \mathbf{p}_{i}}=-2\left(\mathbf{L}_{V_{i}}+\mathbf{R}_{i}-M_{V_{i}} \mathbf{p}_{i}\right),
$$

where

$$
\mathbf{R}_{i}=\sum_{j \in \mathscr{N}_{i}}\left[\frac{1}{2} \mathscr{M}_{i j}\left(\mathbf{C}_{V_{i}}^{T} \mathbf{C}_{V_{i}}-\mathbf{C}_{V_{j}}^{T} \mathbf{C}_{V_{j}}\right)-\mathscr{L}_{i j}\left(\mathbf{C}_{V_{i}}-\mathbf{C}_{V_{j}}\right)\right]
$$

with 


$$
\begin{aligned}
& \mathscr{M}_{i j}=\int_{l_{i j}} \phi(\mathbf{q}) \frac{\partial \mathbf{q}_{l_{i j}}}{\partial \mathbf{p}_{i}} \mathbf{n}_{l_{i j}} d \mathbf{q}, \quad \text { and } \\
& \mathscr{L}_{i j}=\int_{l_{i j}} \phi(\mathbf{q}) \mathbf{q}\left(\frac{\partial \mathbf{q}_{l_{i j}}}{\partial \mathbf{p}_{i}} \mathbf{n}_{l_{i j}}\right)^{T} d \mathbf{q} .
\end{aligned}
$$

The vector $\mathbf{R}_{i}$ is due to the moving boundaries of the Voronoi regions and can be thought as a disturbance to the centroid position.

Collecting these terms together results in

$$
\dot{E}=\sum_{i=1}^{n}\left(-2\left(\mathbf{L}_{V_{i}}+\mathbf{R}_{i}-M_{V_{i}} \mathbf{p}_{i}\right)^{T} \dot{\mathbf{p}}_{i}+F_{i}\right) .
$$

We propose to use the controller

$$
\mathbf{u}_{i}=\frac{\left(\mathbf{L}_{V_{i}}+\mathbf{R}_{i}-M_{V_{i}} \mathbf{p}_{i}\right)}{2\left\|\mathbf{L}_{V_{i}}+\mathbf{R}_{i}-M_{V_{i}} \mathbf{p}_{i}\right\|^{2}}\left(k M_{V_{i}}\left\|\mathbf{C}_{V_{i}}-\mathbf{p}_{i}\right\|^{2}+F_{i}\right) .
$$

With the integrator dynamics $\dot{\mathbf{p}}_{i}=\mathbf{u}_{i}$, this controller gives the result $\dot{E}=-k E$, by substituting (23) back into (22). This means that our controller results in an exponential decay of the performance function

$$
E(t)=E(0) e^{-k t}
$$

There is still a question as to the boundedness of the control signal $\mathbf{u}_{i}$ for the control law (23). We will discuss this point in subsection 3.3.

It is important to emphasize the decentralized aspect of the controller in (23). In order to compute all the terms in (23), each agent relies only on local information. Due to the properties of the intruder functions $\phi_{i}$, previously discussed, each agent only needs to obtain the positions and velocities of intruders that are within a certain tracking distance from the corresponding Voronoi cell. Besides, each agent only needs the centroids of neighbor cells.

\subsection{Computation of Boundary Terms}

There remains a significant practical difficulty in computing the boundary terms $\mathbf{R}_{i}$. We will exploit the geometry of the Voronoi cell to derive a parametric formula for the computation of $\left(\partial \mathbf{q}_{l_{i j}} / \partial \mathbf{p}_{i}\right) \mathbf{n}_{l_{i j}}$. Then computing $\mathscr{M}_{i j}$ and $\mathscr{L}_{i j}$ will be a matter of integrating an explicit formula over a single parameter, and $\mathbf{R}_{i}$ can be readily computed. Define $l_{i j}$ parametrically by

$$
l_{i j}=\left\{\mathbf{q} \mid \mathbf{q}=v_{i j_{1}} \alpha+v_{i j_{2}}(1-\alpha), \quad \alpha \in[0,1]\right\},
$$

where $v_{i j_{1}}, v_{i j_{2}} \in \mathbb{R}^{2}$ are the end points of the line segment $l_{i j}$. Now we can write $\mathbf{q}(\alpha)$ for $\mathbf{q} \in l_{i j}$. By the definition of the Voronoi cell, any point $\mathbf{q}(\alpha)$ must satisfy 


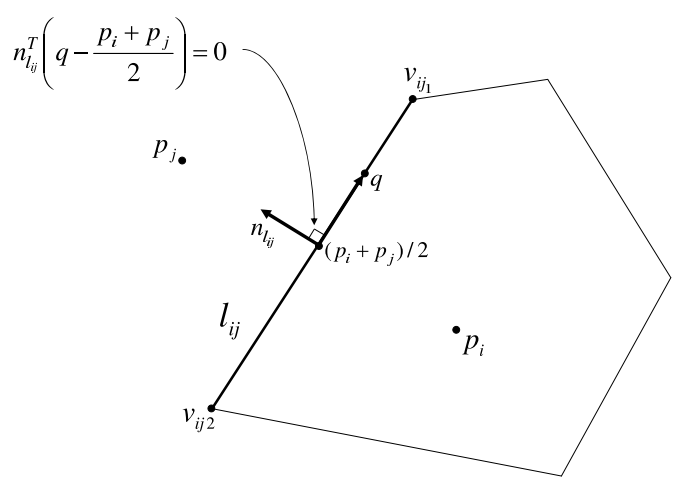

Fig. 1: This figure shows the quantities and constraints associated with the Voronoi boundary shared between neighboring agents.

$$
\mathbf{n}_{l_{i j}}^{T}\left(\mathbf{q}(\alpha)-\frac{\mathbf{p}_{j}+\mathbf{p}_{i}}{2}\right)=0 \quad \forall \mathbf{q} \in l_{i j}
$$

where

$$
\mathbf{n}_{l_{i j}}=\frac{\mathbf{p}_{j}-\mathbf{p}_{i}}{\left\|\mathbf{p}_{j}-\mathbf{p}_{i}\right\|}
$$

Figure 1 shows a schematic of the quantities and constraints associated with the boundary shared between neighboring agents.

Differentiate (26) implicitly with respect to $\mathbf{p}_{i}$ to find the relation

$$
\frac{\partial \mathbf{n}_{l_{i j}}}{\partial \mathbf{p}_{i}}\left(\mathbf{q}(\alpha)-\frac{\mathbf{p}_{j}+\mathbf{p}_{i}}{2}\right)+\frac{\partial \mathbf{q}_{l_{i j}}}{\partial \mathbf{p}_{i}} \mathbf{n}_{l_{i j}}-\frac{\mathbf{n}_{l i j}}{2}=0
$$

where

$$
\frac{\partial \mathbf{n}_{l_{i j}}}{\partial \mathbf{p}_{i}}=\frac{\mathbf{n}_{l_{i j}} \mathbf{n}_{l_{i j}}^{T}-I_{2}}{\left\|\mathbf{p}_{j}-\mathbf{p}_{i}\right\|}
$$

Simplify and substitute using $\mathbf{q}=v_{i j_{1}} \alpha+v_{i j_{2}}(1-\alpha)$ to find the desired formula

$$
\frac{\partial \mathbf{q}_{l_{i j}}}{\partial \mathbf{p}_{i}} \mathbf{n}_{l_{i j}}=\frac{\left(I_{2}-\mathbf{n}_{l_{i j}} \mathbf{n}_{l_{i j}}^{T}\right)\left(\left(v_{i j_{1}}-v_{i j_{2}}\right) \alpha+v_{i j_{2}}-\mathbf{p}_{i}\right)}{\left\|\mathbf{p}_{j}-\mathbf{p}_{i}\right\|}+\frac{\mathbf{n}_{l_{i j}}}{2}
$$

where $I_{2}$ is the $2 \times 2$ identity matrix. Notice (30) is linear in $\alpha$ and can be computed readily given the known quantities $\mathbf{p}_{i}, \mathbf{p}_{j}, v_{i j_{1}}$, and $v_{i j_{2}}$. Then the integrals $\mathscr{M}_{i j}$ and $\mathscr{L}_{i j}$ potentially can be computed analytically over $\alpha \in[0,1]$ if $\phi(\mathbf{q}(\alpha))$ is in a form that allows for this (e.g. if it is polynomial), yielding closed form equations for the boundary terms. If $\phi(\mathbf{q}(\alpha))$ is not in a form for which the integrals can be performed 
analytically, they can be approximated numerically without much computational burden. This gives a method to compute the term $\mathbf{R}_{i}$ (19).

\subsection{Asymptotic Analysis}

In this subsection we address the asymptotic behavior of the control signal $\mathbf{u}_{i}$. One must take care that a singularity does not appear in the control signal as the centroidal configuration is reached. Firstly we formalize the requirement that the environment cannot change too quickly.

Assumption 1 (Bound) $\left\|\int_{V_{i}}\left(2 \mathbf{q}-\mathbf{C}_{V_{i}}-\mathbf{p}_{i}\right) \dot{\phi}(\mathbf{q}, t) d \mathbf{q}\right\| \leq B_{i} \forall i$ and $\forall t$.

This is a design requirement that means that $\phi(\mathbf{q}, t)$ cannot change at an unbounded rate (e.g. the intruders have a bounded velocity).

Now we evaluate the limit to determine the asymptotic behavior of $\mathbf{u}_{i}$

$$
\begin{aligned}
& \lim _{\mathbf{p}_{j} \rightarrow \mathbf{C}_{V_{j}}, \forall j}\left\|\mathbf{u}_{i}\right\|= \\
& \lim _{\mathbf{p}_{j} \rightarrow \mathbf{C}_{V_{j}}, \forall j} \frac{\left\|\mathbf{L}_{V_{i}}+\mathbf{R}_{i}-M_{V_{i}} \mathbf{p}_{i}\right\|}{2\left\|\mathbf{L}_{V_{i}}+\mathbf{R}_{i}-M_{V_{i}} \mathbf{p}_{i}\right\|^{2}}\left|k M_{V_{i}}\left\|\mathbf{C}_{V_{i}}-\mathbf{p}_{i}\right\|^{2}+F_{i}\right| \\
& \quad \leq \lim _{\mathbf{p}_{j} \rightarrow \mathbf{C}_{V_{j}}, \forall j} \frac{k M_{V_{i}}^{-1}\left\|\mathbf{L}_{V_{i}}-M_{V_{i}} \mathbf{p}_{i}\right\|^{2}+\left|F_{i}\right|}{2\left\|\mathbf{L}_{V_{i}}+\mathbf{R}_{i}-M_{V_{i}} \mathbf{p}_{i}\right\|} \\
& \leq \lim _{\mathbf{p}_{j} \rightarrow \mathbf{C}_{V_{j}}, \forall j} \frac{k\left\|\mathbf{L}_{V_{i}}-M_{V_{i}} \mathbf{p}_{i}\right\|^{2}+\left\|\mathbf{L}_{V_{i}}-M_{V_{i}} \mathbf{p}_{i}\right\| B_{i}}{2 M_{V_{i}}\left\|\mathbf{L}_{V_{i}}+\mathbf{R}_{i}-M_{V_{i}} \mathbf{p}_{i}\right\|} \\
& =\lim _{\mathbf{p}_{j} \rightarrow \mathbf{C}_{V_{j}}, \forall j} \frac{\left\|\mathbf{L}_{V_{i}}-M_{V_{i}} \mathbf{p}_{i}\right\| B_{i}}{2 M_{V_{i}}\left\|\mathbf{L}_{V_{i}}+\mathbf{R}_{i}-M_{V_{i}} \mathbf{p}_{i}\right\|}
\end{aligned}
$$

where $B_{i}$ is the bound from Assumption 1 .

Conjecture 0.1 (Singularity).

$$
\left(\mathbf{L}_{V_{i}}-M_{V_{i}} \mathbf{p}_{i}\right) \neq-\mathbf{R}_{i} \quad \forall i \text { and } \forall t \text { except when } \mathbf{C}_{V_{i}}=\mathbf{p}_{i}
$$

Conjecture 0.2 (Finite Limit).

$$
\lim _{\mathbf{p}_{j} \rightarrow \mathbf{C}_{V_{j}}, \forall j} \frac{\left\|\mathbf{L}_{V_{i}}-M_{V_{i}} \mathbf{p}_{i}\right\|}{2 M_{V_{i}}\left\|\mathbf{L}_{V_{i}}+\mathbf{R}_{i}-M_{V_{i}} \mathbf{p}_{i}\right\|} \text { is bounded. }
$$

Remark 0.1. Conjecture 0.1 states that the control signal will never become unbounded in finite time due to a cancellation of vectors in the denominator. It would be difficult to prove whether our system has this property a priori for a given set of initial conditions, but one can easily enforce a saturation bound on the control input to avoid this problem. Indeed, we do not see this problem occurring in simulation or hardware experiments. 
Remark 0.2. Conjecture 0.2 states that the control input does not blow-up asymptotically as an agent converges to its centroid. One straightforward way to prove this conjecture would be to show that $\mathbf{R}_{i}$ does not converge to zero. Unfortunately, it can be proved that for bounded $\mathscr{M}_{i j}$ and $\mathscr{L}_{i j}$

$$
\mathbf{p}_{i}=\mathbf{C}_{V_{i}} \quad \forall i \quad \text { implies } \quad \mathbf{R}_{i}=0 \quad \forall i
$$

Again, we were unable to prove this conjecture, but we rely upon simulations and experiments which show that the input does not blow-up. In practice one can impose saturation constraints to prevent this from happening.

\section{Numerical Simulations}

In this section we present ideal simulations that verify the proposed approach. The simple model in (10) is used. In these simulations and all the other simulations and experiments presented in this paper, we used parameters $\alpha_{i}=50, \beta=1$, and Gaussian density function (eq. (14)) with parameters $\sigma=0.5, A=0.64$. We implemented the controller in (23) which is related to the Lyapunov function in (15). Figs. 2a, 2b, and $2 \mathrm{c}$ present the initial configuration, executed path, and final configuration, respectively, for a simulation with a team of five robots and one intruder. The intruder moves with constant speed of $0.1 \mathrm{~m} / \mathrm{s}$ in a uniform circular motion centered at the origin with unitary radius. Figs. $2 d, 2 e$, and $2 \mathrm{f}$ present simulation results with five robots and two intruders. The second intruder moves with constant velocity vector $[0,0.1]^{T}$. In both cases we used $k=40$. A more complicated scenario is shown in Figs. $2 \mathrm{~g}, 2 \mathrm{~h}$, and $2 \mathrm{i}$ where we have a team of nine robots and three intruders. Once again, one intruder moves according to the uniform circular motion. The other two intruders move with constant velocity vectors $[0,-0.05]^{T}$ and $[-0.05,0]^{T}$. In this last simulation we used $k=20$.

The Lyapunov function exponential decay is verified in Figures $2 \mathrm{j}, 2 \mathrm{k}$, and 21 where we also show the bound given in (24). One can observe that the Lyapunov functions follow the expected bound as desired, even though we noticed some "jumps" in some simulations as in Fig. 21. These "jumps" were caused by numerical errors related to the resolution in the numerical computation of integrals and also the simulation time step used. In fact, we verified that the system is very sensitive to these numbers.

\section{Experiments}

In this section, we verify the proposed approach in Gazebo [6], a simulated robotic environment, and on actual robots. These experiments were based on five Scarabs, 


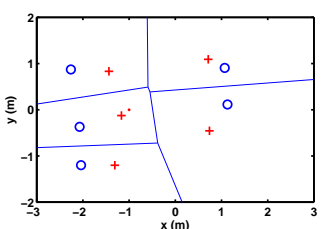

(a)

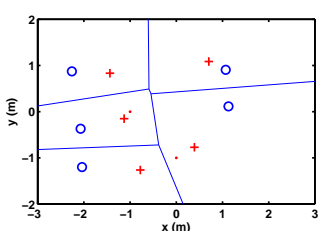

(d)

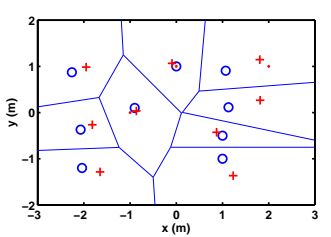

(g)

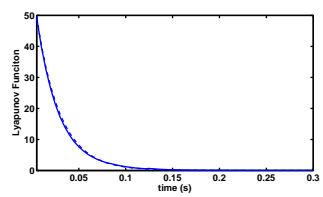

(j)

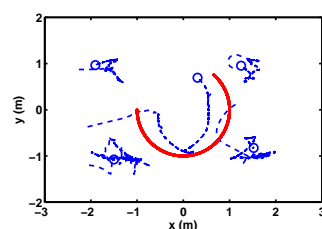

(b)

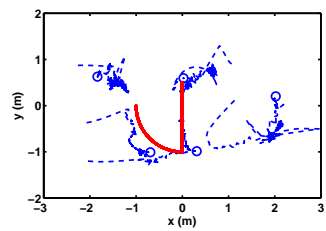

(e)

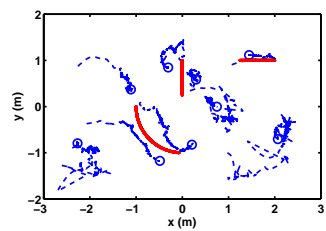

(h)

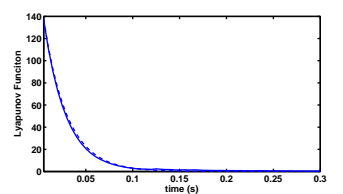

(k)

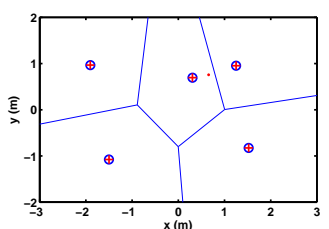

(c)

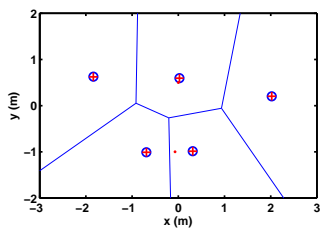

(f)

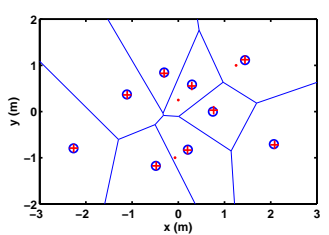

(i)

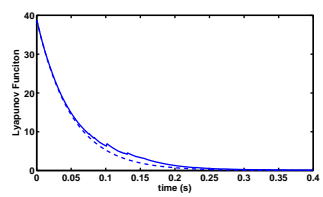

(1)

Fig. 2: Simulation results for three scenarios: (i) five robots and one intruder, $k=$ 40, (Figs. 2a, 2b, 2c, and 2j), (ii) five robots and two intruders, $k=40$, (Figs. 2d, $2 \mathrm{e}, 2 \mathrm{f}$, and $2 \mathrm{k}$ ), (iii) nine robots and three intruders, $k=20$, (Figs. $2 \mathrm{~g}, 2 \mathrm{~h}, 2 \mathrm{i}$, and $21)$. The positions of the agents are given by the circles while the positions of the intruders are given by the dots. The crosses show the positions of the centroids. For each scenario it is shown initial configuration, trajectories, final configuration, and Lyapunov function evolution (solid line) along with the theoretical bound in (24)(dashed line). 


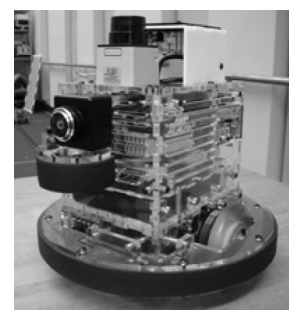

Fig. 3: Scarab, differential drive robotic platform, outfitted with a camera, Urg laser unit and tracking beacon.

differential drive robots, shown in Figure 3. For more information refer to [8]. We assumed an $6 \mathrm{~m} \times 4 \mathrm{~m}$ environment.

The first scenario is the one presented in the last section with five robots and one intruder. The initial configuration of the robots are the same as in Figure 2a. First, we present the Gazebo simulation results. In Figures $4 a$ and $4 b$, the final configuration of the robots are given along with the Lyapunov function $E$. One can see that this function is not bounded by the theoretical bound in (24) when $k=40$. This inconsistency with the theoretical results comes from many factors including the saturation of the acceleration caused by the limitations of a simulated robot, the nonholonomic constraints, and the repulsion term added to $\mathbf{u}_{i}$ to avoid collisions:

$$
\mathbf{u}_{i, \text { expt }}=\mathbf{u}_{i}+\sum_{i \neq j} \frac{\kappa}{\left\|\mathbf{p}_{i}-\mathbf{p}_{j}\right\|^{2}} \mathbf{n}_{l j i}
$$

where $\kappa$ is a small positive collision avoidance gain.

The experiment was conducted using actual robots. Each robot localizes using an overhead tracking system and velocity information from its motor controllers. Their pose and velocities are sent to a single computer running Player [6], which calculates the new controls and sends these commands to the individual robots. Each robot is kinematically controlled using feedback linearization.

In Figures $4 \mathrm{c}$ and $4 \mathrm{~d}$, the results of the robot experiment are presented. We observed the same decay seen in Gazebo simulations as shown in Figure $4 \mathrm{~b}$ along with the inconsistency with the theoretical bound.

We also conducted gazebo simulations for the scenario where we have nine robots and three intruders. The trajectories and configurations for different instants of time are presented in Figs. 5a, 5b, and 5c. Fig. 5d presents the Lyapunov function evolution. As expected, once again the exponential decay does not match the bound, with $k=20$ in this case. 


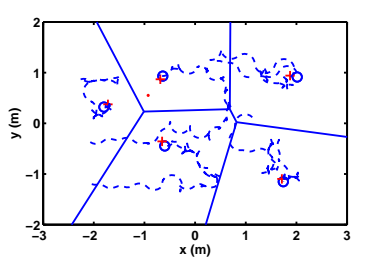

(a)

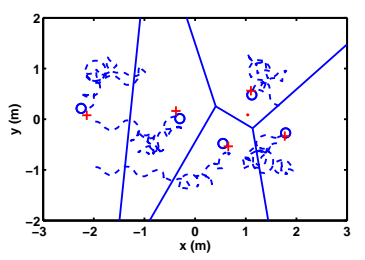

(c)

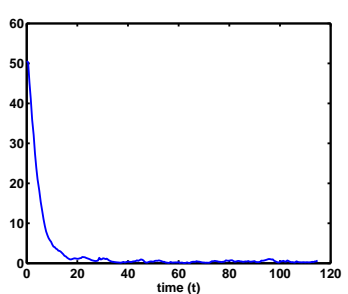

(b)

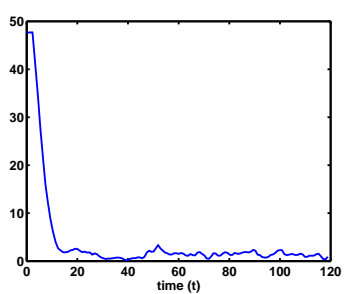

(d)

Fig. 4: Results for one moving intruder and five robots. Fig. 4a The final configuration and trajectories for a Gazebo simulation. Fig. 4b Evolution of the Lyapunov function for the Gazebo simulation. Figs. $4 \mathrm{c}$ and $4 \mathrm{~d}$ Corresponding results for actual robots experiment.

\section{Conclusion}

This work proposes an extension of the framework proposed in [3] to solve the problem of tracking multiple intruders in a pre-specified region. The basic subtasks: task assignment, coverage, and tracking are coupled in an elegant manner. The proposed decentralized controller guarantees exponential convergence. A complete analysis of the singularities that are present in the controller is difficult. However, after conducting simulations and experiments we believe that such singularities do not pose a problem in practice.

Acknowledgements We gratefully acknowledge support from NSF grant IIS-0427313, ONR grant N00014-08-1-0696, ARO grants W911NF-05-1-0219 and W911NF-08-2-0004, and CNPqBrazil.

\section{References}

1. J. Corté, S. Martínez, and F. Bullo. Spatially-distributed coverage optimization and control with limited-range interactions. ESIAM: Control, Optimisation and Calculus of Variations, 


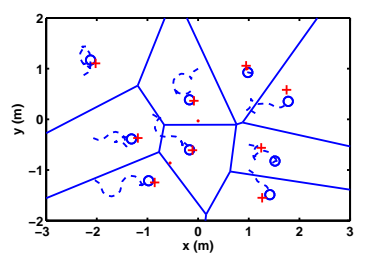

(a)

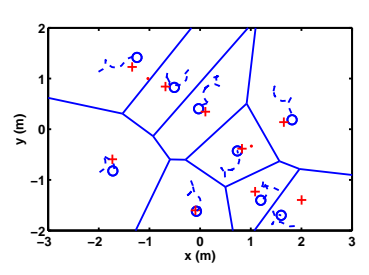

(c)

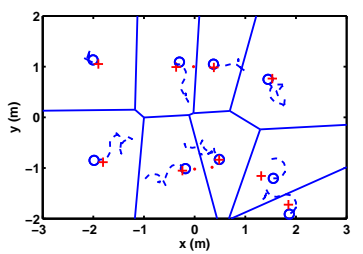

(b)

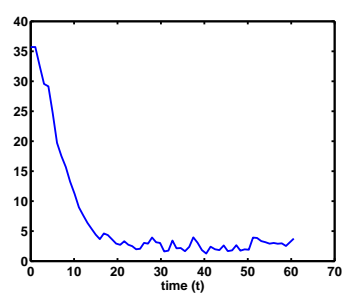

(d)

Fig. 5: Results for three moving intruders and nine robots. Figs. 5a,5b, and 5c show trajectories for $t=0.00$ to $20.20 \mathrm{sec}, t=20.20$ to $40.44 \mathrm{sec}$, and $t=40.44$ to 60.60 sec. Fig. 5d Evolution of the Lyapunov function for the Gazebo simulation.

11:691-719, 2005.

2. J. Cortés, S. Martínez, T. Karatas, and F. Bullo. Coverage control for mobile sensing networks: Variations on a theme. In Proceedings of the 10th Mediterranean Conf. on Control and Automation, pages 1-9, Lisbon, Portugal, 2002.

3. J. Cortés, S. Martínez, T. Karatas, and F. Bullo. Coverage control for mobile sensing networks. IEEE Transactions on Robotics and Automation, 20(2):243-255, April 2004.

4. Z. Drezner. Facility Location: A Survey of Applications and Methods. Springer Series in Operations Research. Springer-Verlag, New York, 1995.

5. H. Flanders. Differentiation under the integral sign. American Mathematical Monthly, 80(6):615-627, 1973.

6. B. Gerkey, R. T. Vaughan, and A. Howard. The Player/Stage project: Tools for multi-robot and distributed sensor systems. pages 317-323, 2003.

7. S. Lloyd. Least squares quantization in PCM. IEEE Trans. Inform. Theory, 28(2):129-137, 1982.

8. N. Michael, J. Fink, and V. Kumar. Experimental testbed for large multirobot teams. IEEE Robotics and Automation Magazine, 15(1):53-61, 2008.

9. A. Okabe, B. Boots, K. Sugihara, and S. N. Chiu. Spatial Tessellations: Concepts and Applications of Voronoi Diagrams. Wiley Series in Probability and Statistics. New york: Wiley, 2nd edition, 2000.

10. L. C. A. Pimenta, V. Kumar, R. C. Mesquita, and G. A. S. Pereira. Sensing and coverage for a network of heterogeneous robots. In Proc. 47th IEEE Conf. on Decision and Control, pages 3947-3952, Cancun, Mexico, 2008.

11. M. Schwager, J.-J. E. Slotine, and D. Rus. Consensus learning for distributed coverage control. In Proc. IEEE Int. Conf. Robot. Automat., pages 1042-1048, Pasadena, USA, 2008.

12. A. Weber. Theory of the Location of Industries. The University of Chicago Press, Chicago, IL, 1929. Translated by Carl. J. Friedrich. 